\title{
The efficacy of ATP removal on gym contact surfaces using disinfectant wipes
}

\author{
Victor Tam ${ }^{1}$, Bobby Sidhu ${ }^{2}$ \\ 1 Lead Author, School of Health Sciences, British Columbia Institute of Technology, Burnaby, BC \\ 2 Supervisor, School of Health Sciences, British Columbia Institute of Technology, Burnaby, BC
}

\begin{abstract}
Background: Gym equipment surfaces are known to harbor a range of contaminants due to the wide range of community use of the equipment. Certain gym equipment undergoes daily sanitation, however many other equipment surfaces do not. This study measures the levels of contamination on certain gym equipment surfaces at an educational institute gym facility and determines the contamination levels after disinfectant wipes are applied.

Methods: The method to obtain the data was determined by the use of the Hygiena Systemsure II ATP analyzer in conjunction with Hygiena Ultrasnap ATP surface swabs. Gym equipment (barbells, dumbbells, machine handles, cable attachments) and other surfaces (benches, floor mats) were swabbed subsequently after a random gym patron had used the equipment to capture an accurate representation of the cleanliness of the surfaces. Disinfectant wipes were then applied to the same area before being swabbed again to determine contamination levels after disinfection.

Results: The results demonstrated a statistically significant difference in the reduction of ATP levels with the use of disinfectant wipes with a $p$-value of 0.00001 at $\alpha=0.05$. Alpha error was highly unlikely with a p-value being that low. Power was $99.9 \%$, therefore there is a strong likelihood that we are correctly rejecting the null hypothesis.

Conclusion: The study can conclude that disinfectant wipes do make a significant difference in surface cleanliness levels. Equipment that does not undergo routine cleaning such as the equipment used by the hands carry a much higher contamination rate than the body contact surfaces. Gym patrons should disinfect all body contact surfaces prior to use to reduce the risk of getting an infectious disease.
\end{abstract}

Keywords: Gym, disinfection, Ultrasnap, hygiene, ATP, RLU,

\section{INTRODUCTION}

Gym sanitation is widely ignored by the majority of patrons who enter any facility. Workout equipment, such as barbells, dumbbells and benches are touched over and over throughout the day between different users; through each contact, skin and sweat residues left behind. Sweat and skin cells that are left behind can contain potentially harmful bacteria. Consequently, the human hands play a major role in disease transmission. The fecal-oral route is one of the many ways diseases are spread. Improper hand washing following the use of the bathroom could lead to the person contaminating various things. The Canadian Center for Occupational Health and Safety (2011) states hand washing is the single most effective way to prevent the spread of infection. Gym staff members expect patrons to abide by policies and clean and wipe down equipment after use. Cleaning compounds such as sanitizers are supplied on gym floors to disinfect equipment but are not often used. Hence, there is a potential for of disease to spread if proper sanitation is not met due to harmful pathogens contaminating the surfaces. Emerging diseases these days can occur anytime and prevention through control measures are adamantly required. Standard hygiene practices are encouraged to prevent the spread of infections. 


\section{PURPOSE OF STUDY}

The purpose of this study is to evaluate the efficacy of disinfectant wipes on gym contact surfaces. To conduct this study, contact surfaces was swabbed immediately after being used without sanitizing as well as swabbed after sanitizing to compare the difference in sanitation levels. The tool used in the evaluation of sanitation levels is an ATP bioluminescence analyzer. More information on this instrument will be covered in the methods section of this study.

The research question, $\mathrm{H}_{\mathrm{o}} / \mathrm{H}_{\mathrm{a}}$ as follows:

$\mathrm{H}_{0}$ : ATP levels (in RLUs) after disinfection $>=$ than ATP levels before disinfection

$\mathrm{H}_{\mathrm{a}}$ : ATP levels (in RLUs) after disinfection $<$ than ATP levels before disinfection

\section{LITERATURE REVIEW}

\section{Etiological agents}

Gym facilities can harbor many microorganisms that may be harmful if introduced into the human system through open wounds. By in large, athletes are more at risk due to the variety of stresses their skin undertakes. Injuries and wounds are likely to develop on the skin due to rough contact sports. These small cuts can then lead to skin infections caused by bacteria, viruses, or fungi which can lead to contagious outbreaks if proper care is not taken (Decker, 2010). For example, athlete's foot, a fungal infection around the feet, is most commonly spread from contaminated surfaces due to improper cleaning and sanitation of shower facilities. Jock itch, herpes simplex, plantar warts, palmer warts, impetigo and ring worms are also infections of concern. Consequently, these infections can also be a nuisance and inhibit an athlete from competing in an exhibition or event. Some of these infections can also be life-threatening if left untreated.

Skin and soft tissue infections (SSTIs) are a common problem for people. These infections are commonly caused by Staphylococcus aureus, a ubiquitous pathogen commonly found on the skin and in the nasal canal of carriers. The bacterium normally is not pathogenic but can cause skin lesions if the opportunity presents itself through an open wound or abrasion. Normal Staph infections can become serious if left untreated but can be managed with antibiotics. More troublesome pathogens include methicillin-resistant $S$. aureus (MRSA). This pathogen gained antibiotic resistance in the health care settings through constant exposure to antibiotics. Standard beta-lactam antibiotic are ineffective against the pathogen, making it more difficult to treat. Different strains of this pathogen have emerged into the community settings and are commonly referred to now as community-associated MRSA (CA-MRSA). Therefore, carriers of CA-MRSA can pose a risk to other gym patrons if they do not properly disinfect gym equipment for others.

\section{Emergence of CA-MRSA}

The CDC (2013) suggests that approximately 29\% and $1.5 \%$ of people are carriers of $S$. aureus and MRSA respectively. Skin infections are extremely common around the world. There are approximately 12 million Americans who visit their physicians to be examined for MRSA infections (Stanforth, Krause, Starkley \& Ryan. 2010). Rockwell et al. says in British Columbia, CA-MRSA was considered rare but numerous cases have been reported over the past decade (2005). Furthermore, northern Manitoba had a rapid emergence of cases in the years between 2003 and 2006 (Larcombe, Waruk, Schellenberg, \& Ormond, 2007). Hospital-associated MRSA (HAMRSA) strains have different anti-biograms than community-associated MRSA due to unique molecular characteristics (Skov et al., 2012; Miller, \& Diep, 2008). HA-MRSA infections often require a cocktail of non-beta-lactam antibiotics to treat whereas multidrug resistance in CA-MRSA is rare. The CDC (2013) created standardized case definition for CA-MRSA which is an MRSA infection in a person who has none of the factors that came from HA-MRSA, including isolation of MRSA more than 48 hours after any hospital admission, surgery, dialysis, percutaneous device, or previous isolation of MRSA.

\section{Risks associated with CA-MRSA}

CA-MRSA disease outbreaks have been seen among children, homosexual men having sex, athletes, and military personnel, and inmates (Miller, \& Diep, 2008; Cohen, 2008). Community-associated MRSA 
skin infection legions are pleomorphic that account for approximately 90\% of bacterial SSTIs (Cohen, 2008; Skov et al., 2012). These skin lesions usually manifest into cellulitis and pus filled abscesses or boils that require incision and drainage used in combination with strong prescription antibiotics for sufficient treatment. Cases are generally mild if treated appropriately within a timely manner but can develop into more severe life-threatening cases such as necrotizing fasciitis, necrotizing pneumonia or sepsis (Skov et al., 2012; Miller, \& Diep, 2008). These infections should be treated and bandaged properly as a control measure to prevent any further contamination. CA-MRSA infections are noted to recur in patients and potentially spread to other household members (Miller, \& Diep, 2008). CAMRSA has the tendency to colonize on household objects, such as door knobs, toilet handles, and kitchen sinks. MRSA has also been reported to be able to penetrate undamaged skin, infecting deep layers of tissues (Shukla, 2006). Multiple studies have identified MRSA reservoirs present on wrestling mats, in showers, bathrooms, and locker rooms (Tonn, \& Ryan, 2013). Most people who contract a CA-MRSA skin infection believe they got a spider-bite, however never seeing an actual spider bite them (Decker, 2010). In a New York Times (2010) article, a 21-year old wrestling athlete contracted CA-MRSA from an exhibition match. What he thought was a small pimple manifested into a potentially lethal CA-MRSA infection. The transmission was either through direct skin-to-skin contact from a contaminated person or through indirect contact of a contaminated surface such as the wrestling mat. That being said, studies show that CAMRSA is more commonly spread through person-toperson contact rather than indirect contact of contaminated fomites (Miller, \& Diep, 2008). Items such as razors, bath towels, and wrestling headgear have been implicated to harbour MRSA, making it dangerous to share personal belongings. Even bar soap, workout benches, body suits and whirlpools have been implicated in causing CA-MRSA infections (Miller,\& Diep, 2008).

Despite all these studies implicating the transmission of MRSA, a study by Ryan et al. suggested that gym surfaces were not a source or reservoir for MRSA (2011). The study supported that gym facilities do not require a vigorous cleaning regiment and that community-associated transmission of MRSA is more likely caused from skin-to-skin contact rather through skin-to-contaminated surface. In their methodology, swabs were used that were selective for potential isolates or MRSA and MSSA (methicillin-susceptible Staph aureus). A total of 240 samples were swabbed and cultured but found no presence of MRSA or MSSA on any of the common gym contact surfaces such as benches, barbells, dumbbells and treadmills. Limitations of this study can arise from the fact that the patrons in the gym do not harbour any MRSA or if the gym facility surfaces were adequately sanitized prior to samples being collected. Furthermore, broth enhancement was also not used during swab isolation which could have affected the growth sensitivity of any pathogens. The gyms were also supplied with disinfectant wipes and had policies for standard cleaning and maintenance. It is very possible that disinfecting wipes were effective in reducing or eliminating any bacterial colonization on the gym surfaces or that MRSA actually cannot survive on gym surfaces. Whether or not any of these studies hold true, CA-MRSA is an emerging pathogen affecting healthy individuals and control measures should be put in place to safeguard the community from this pathogen. Taking this into context of the study, it is still conceivable that gym equipment surfaces can be contaminated from an infected person making it a possible medium for indirect transmission.

\section{Influenza}

The influenza virus is a respiratory illness caused by a virus, commonly known as the flu. The flu comes in seasons and is highly contagious. Symptoms of the flu include fever, cough, sore throat, runny or stuffy nose, muscle aches, headaches, and the feeling of fatigue. People are capable of spreading the flu before an onset of the symptoms by one day, and during the sickness, making communicability dangerously high (CDC, 2013b). Infected people are advised to stay indoors until they are fully rested to prevent the spread of the flu. The potential to spread the influenza virus is highly possible if an infected person attends a gym facility prior to feeling the onset of symptoms. A simple cough can aerosolize the virus and infect people up to 6 feet (CDC, 2013b). Infected people can also contaminate surfaces through coughing on the hands followed by touching the surfaces making the object a fomite of infection to others.

\section{Guidelines on prevention}

Currently there is no legislation or regulations in Canada with regards to sanitation and cleanliness of gym establishments. Health inspectors also do not 
play a role in inspecting fitness facilities unless there are implications of a hazardous outbreak. With that being said, the National Athletic Trainers' Association (NATA) released detailed guidelines for prevention, recognition, and management of the common SSTIs (Zinder et al., 2010). These guidelines were based on current research and literature. If outbreaks do occur in the future, these guidelines can aid in controlling the spread of CAMRSA infections among healthy communities.

\begin{tabular}{ll}
\hline $\mathbf{1}$ & Structural maintenance must be adequate to limit the spread of infectious agents \\
\hline $\mathbf{2}$ & A clean environment must be maintained in the athletic training facility, locker rooms, and all athletic \\
& venues \\
$\mathbf{3}$ & Health care physicians and athletes should follow good hygiene practices \\
$\mathbf{4}$ & Use alcohol-based antiseptics for hands if hand-wash station unavailable \\
$\mathbf{6}$ & Athletes with open wounds, scrapes, or scratched must avoid whirlpools and common tubs \\
$\mathbf{7}$ & Athletes are to report all abrasions, cuts, and skin lesions to and to seek medical help for proper \\
& cleansing, treatment, and dressing
\end{tabular}

(Zinder et al., 2010)

The CDC recommends that shared equipment that comes in direct contact with skin should be disinfected and properly dried after each use (CDC, 2013a). The type of disinfectant or sanitizer should be EPA-approved with active ingredients that are capable removing harmful microorganisms such as CA-MRSA. Cleaners such as

\section{MATERIALS USED}

A list of the following materials used in this study are listed below in Table 1

Table 1: Materials used chlorine or bleach solutions can be toxic if used in high concentrations therefor precautions should be taken to ensure safe levels are being used. Other chemical sanitizers will have instruction labels listed and should be followed to ensure proper use of the disinfectant.

\begin{tabular}{|c|c|}
\hline Item & Description \\
\hline $\begin{array}{l}\text { Hygiena SystemSURE IITM ATP Hygiene Monitoring } \\
\text { System (Hygiena, 2005a) }\end{array}$ & $\begin{array}{l}\text { A palm-sized instrument that measures adenosine } \\
\text { triphosphate (ATP) molecules }\end{array}$ \\
\hline $\begin{array}{l}\text { Ultrasnap }^{\mathrm{TM}} \text { ATP Surface Test } \\
\text { (Hygiena, 2005b) }\end{array}$ & $\begin{array}{l}\text { A pen-shaped swab containing unique liquid-stable } \\
\text { reagent that picks up indicative organic matter off } \\
\text { surfaces for monitoring; used with the Hygiena } \\
\text { SystemSURE II }{ }^{\mathrm{TM}} \text { instrument }\end{array}$ \\
\hline $\begin{array}{l}\text { Calibration control kit } \\
\text { (Hygiena, 2005c) }\end{array}$ & $\begin{array}{l}\text { Calibration control swab kits consisting of a positive and } \\
\text { negative rod to verify calibration of Hygiena } \\
\text { SystemSURE IITM instrument }\end{array}$ \\
\hline Various gym equipment surfaces & $\begin{array}{l}\text { Gym equipment surfaces being swabbed such as } \\
\text { barbells, dumbbells, machine handles, and benches; }\end{array}$ \\
\hline
\end{tabular}


where most hand contact and sweat residues reside

Certainty $^{\mathrm{TM}}$ Brand Disinfectant Wipes

(Lalema, 2013)

\section{Gloves}

Microsoft Excel 2010 Data Analysis package

NCSS 9 Statistical Analysis software

(Hintz, 2013)
Disinfectant wipes, provided by BCIT gym facility; used

to wipe gym contact surfaces to monitor hygiene levels

after initial swabbing

Used to handle materials aseptically

Used for descriptive statistical analysis of data

Used for inferential statistical analysis of data

\section{Hygiena SystemSURE II ${ }^{\mathrm{TM}}$ ATP Hygiene Monitoring System}

Adenosine Triphosphate (ATP) Bioluminescence assay is a technology that provides rapid fast analysis of surfaces for cleanliness. These instruments are becoming widely accepted in the food industry as a method of monitoring sanitation. ATP is the basic building block of energy for all biological organisms including microbes. These ATP bioluminescence instruments are able to detect levels of ATP on a surface using an enzyme (luciferase) and substrate (luciferin), found in fireflies. The amount of light generated from the swab sample is directly proportional to the amount of ATP present in the sample. The assay is set up so that ATP levels are then measured with a linear relationship in Relative Light Units (RLUs). The higher the number of the result, the more contamination is present on the surface that was swabbed. These tests are extremely rapid and can give results within 15 seconds whereas the traditional aerobic plate count (APC) method can take up to 48 hours to yield results. The instrument is highly sensitive and can detect down to 1 femtomole of ATP. ATP testing however does not test or yield any other informational data other than the presence of ATP residue. Specific qualitative data such as microorganisms, yeasts and molds will be accurately represented with aerobic plate counting.

\section{Ultrasnap $^{\mathrm{TM}}$ ATP Surface Test}

The Ultrasnap ${ }^{\mathrm{TM}}$ ATP surface testing swabs work in conjunction with the Hygiena SystemSURE II ${ }^{\mathrm{TM}}$ ATP Hygiene monitoring system to provide accurate results of ATP levels on surface samples represented in RLU. These pen-sized swabs contain a liquidstable reagent with a pre-moistened swab, containing a cationic agent to release any ATP residues left from any intact cells. When sampling, ATP residues will be accurately represented in the swab.

\section{Certainty $^{\mathrm{TM}}$ Brand Disinfectant Wipes}

Certainty ${ }^{\mathrm{TM}}$ brand disinfectant wipes are thick, strong, pre-moistened towels that are alcohol free with a natural fragrance. They are safe on the hands but may cause irritation for some sensitive people (Lalema, 2013). The active ingredients in these wipes are alkyl dimethyl ethylbenzyl ammonium chloride and benzalkonium chloride, both a strength of 0.025\%. The Environmental Protection Agency (EPA) has approved of these active ingredients as a disinfectant for multiple surfaces, including for the elimination of Methicillin-resistant Staphylococcus aureus (Environmental Protection Agency, 2012). The wipes are Health Canada approved as a technical disinfectant: DIN \#: 02325853 (Health Canada, 2009).

\section{Standard Procedure}

The study was conducted at the British Columbia Institute of Technology Fit Pit gym facility. Prior to sampling, the author wore gloves to prevent any contamination of the equipment and swabs.

Swabs were labeled respectively to pieces of equipment to be sampled. When gym patrons were finished with certain pieces of equipment, permission was asked to use that equipment to be sampled for. Using the sterile Ultrasnap swabs, a four by four area, representing where the most hand or body contact has touched, was swabbed for. The enumerated swab was then inserted into the Hygiena SystemSURE II instrument to determine the level of hygiene of the surface. After waiting 15 seconds for the instrument to analyze the results, the data was then recorded. Using the Certainty brand disinfectant wipes that were provided at the gym facility, the same area that was previously sampled was wiped down. The surfaces were then allowed to air dry before proceeding to being sampled for. Following the same 
previous procedure, the same area was swabbed for in a four by four area. Results were then determined

\section{Justification for Method}

Due to time constraints and the budget for the experiment, this method was more suitable over the other alternatives listed below. The Hygiena SystemSURE II TM provides an accurate representation of surface hygiene in a short amount of time. Results can be given in 15 seconds whereas the other methods can take up to days. Microbial load of the surfaces are represented in ATP levels and displayed in RLUs linearly. A study by Leon, \& Albrecht suggests that there is a strong correlation between the approved standard of aerobic plate counting and ATP bioluminescence assays within ranges between $10^{3}-10^{7} \mathrm{cfu} / \mathrm{ml}$, representative of its accuracy (2007).

\section{Alternate Methods}

3M TM Petrifilm Rapid Coliform Count Plates count are an alternative method for testing the hygiene of the gym equipment surfaces (3M Microbiology, 2013). This method will provide a quantitative representation in total coliforms of the surface being sampled. Samples must be collected using $3 \mathrm{M}^{\mathrm{TM}}$ Quick Swabs and then plated onto the $3 \mathrm{M}^{\mathrm{TM}}$ Petrifilm Rapid Coliform Count Plates. This method is quick and will give results within 4-24 hours, however, requires incubation after inoculation to produce accurate results (3M Microbiology, 2013).

RODAC (Replicate organism detection and counting) plates may also be used for the detection and enumeration of microorganisms. RODAC plates are suitable for collecting samples from identical areas before and after treatment to monitor cleanliness of the sampled surface (BD Prepared Media, 2013). A problem with this method is that some of the surfaces being sampled are not flat, thus making collection efficacy an issue for RODAC plates. A study suggests that collection efficiency is poorer on dry using the same instrument and recorded after.

surfaces and can improve if surfaces were moist (Pinto, 2009) Also the overall surface areas of RODAC plates are small and only capture a portion of the representative sample.

\section{Reliability and Validity of Measures}

Hygiena SystemSURE II ${ }^{\mathrm{TM}}$ ATP Hygiene monitoring system is a proven instrument that provides reliable results on hygiene of a surface. The instrument is very sensitive, making reproduction of exact results unlikely in repeated samples. This can lead to slight variations in the end results. Compared to other commercial ATP hygiene monitoring systems, the Hygiena brand system provided the most precise, accurate and repeatable data (Hygiena, n.d.). The author will be using the same instrument and following the exact procedures in a consistent fashion to increase reliability. The instrument also internally self-calibrates, removing the possibility of human error in manual calibration. There is low internal validity due to the lack of control of the public's use of the gym equipment such that every person's hygiene is different. The author is well trained in swabbing aseptically through accreditation in quality control and quality assurance courses taken at Camosun College through the Applied Chemistry and Biotechnology program. The researcher will be performing the swabbing and analysis to increase reliability through similar swabbing technique.

\section{Calibration of Instruments}

The Hygiena SystemSURE II ${ }^{\mathrm{TM}}$ ATP hygiene monitoring system performs its own internal selfcalibration check upon operation. For further confirmation in calibration, calibration control kits are supplied by the manufacturer with specified positive and negative control swabs (Hygiena, 2005c). The unit is fully functional and working in proper specifications after calibration.

\section{Inclusion and Exclusion Criteria}

\begin{tabular}{ll}
\hline Inclusion & Exclusion \\
\hline BCIT gym facility & Other gym facilities \\
\hline
\end{tabular}


Dumbbell handles, Barbell handles, Machine handles

\section{Benches, Floor mats}

\section{Ethical considerations}

No ethical considerations are applicable to this study because it is not a human based study or a survey $(\mathrm{H}$. Heacock, Personal communication, 2013 Nov 5).

\section{Pilot Study}

A pilot study was performed to increase the specificity and validity of this study. The lab technician (F. Shaw) calibrated the instrument and conducted calibration swabs to test the reliability and validity of the instrument prior to the actual study being performed.

\section{Statistical analysis}

\section{Hypothesis}

$\mathrm{H}_{\mathrm{o}}$ : ATP levels (in RLUs) after disinfection $>=$ than ATP levels before disinfection

Table 2: Descriptive Statistics of Raw Data

\begin{tabular}{|l|r|}
\hline \multicolumn{2}{|c|}{ Hand Contact Surfaces Not Wiped in RLU } \\
\hline & \\
\hline Mean & 330.4444444 \\
\hline Standard Error & 54.00642798 \\
\hline Median & 281.5 \\
\hline Mode & \#N/A \\
\hline Standard Deviation & 229.1298687 \\
\hline Sample Variance & 52500.49673 \\
\hline Range & 796 \\
\hline Minimum & 59 \\
\hline Maximum & 5948 \\
\hline Sum & 18 \\
\hline Count & \\
\hline
\end{tabular}

$\mathrm{H}_{\mathrm{a}}$ : ATP levels (in RLUs) after disinfection $<$ than ATP levels before disinfection

A one-sided paired t-test is performed to assess the difference in ATP levels between the variables of surfaces being not wiped with disinfectant and wiped with disinfectant.

\section{Descriptive Statistics}

The raw data collected for analysis was numerical and discrete. Microsoft Office Excel 2010 was used to categorize separate four variables of the data; hand contact surfaces not wiped and wiped with disinfectant wipe, and body contact surfaces not wiped and wiped with disinfectant wipes. The groupings between hand contact surfaces and body contact surfaces showed a trend in different results. The data analysis package from Microsoft Excel 2010 was used to form the descriptive statistics listed below.

\begin{tabular}{|l|r|}
\hline \multicolumn{2}{|c|}{ Hand Contact Surfaces Wipe in RLU } \\
\hline Mean & 112.7777778 \\
\hline Standard Error & 22.80172502 \\
\hline Median & 71 \\
\hline Mode & 70 \\
\hline Standard Deviation & 96.73952629 \\
\hline Sample Variance & 9358.535948 \\
\hline Range & 354 \\
\hline Minimum & 9 \\
\hline Maximum & 2030 \\
\hline Sum & 18 \\
\hline Count & \\
\hline
\end{tabular}




\begin{tabular}{|l|r|}
\hline \multicolumn{2}{|c|}{ Body Contact Surfaces Not Wiped in RLU } \\
\hline & \\
\hline Mean & 113.1176471 \\
\hline Standard Error & 27.98172754 \\
\hline Median & 69 \\
\hline Mode & 136 \\
\hline Standard Deviation & 115.3716182 \\
\hline Sample Variance & 13310.61029 \\
\hline Range & 437 \\
\hline Minimum & 2 \\
\hline Maximum & 439 \\
\hline Sum & 1923 \\
\hline Count & 17 \\
\hline
\end{tabular}

From the data collected, hand contact surfaces were seen to be the highest compared to body contact surfaces with an average mean of 330 RLUs versus 113 RLUs right after use of the equipment. The standard deviations for the both hand and body contact surfaces non-wiped resulted in a value of 229 RLUs and 115 RLUs respectively. Subsequent disinfectant values demonstrated a larger reduction for both hand and body contact surfaces with an

\begin{tabular}{|l|r|}
\hline \multicolumn{2}{|c|}{ Body Contact Surfaces Wiped in RLU } \\
\hline Mean & 25.23529412 \\
\hline Standard Error & 6.062498885 \\
\hline Median & 17 \\
\hline Mode & 0 \\
\hline Standard Deviation & 24.99632326 \\
\hline Sample Variance & 624.8161765 \\
\hline Range & 83 \\
\hline Minimum & 83 \\
\hline Maximum & 429 \\
\hline Sum & 17 \\
\hline Count & \\
\hline
\end{tabular}

average mean value of 112 RLUs and 25 RLUs respectively. Standard deviations for both hand and body contact surfaces after disinfection resulted in values of 97 RLUs and 25 RLUs respectively. The figures below represent the groups of data (hand contact surfaces and body contact surfaces) showing the difference in RLUs for the specific equipment tested, before and after using a disinfectant wipe.

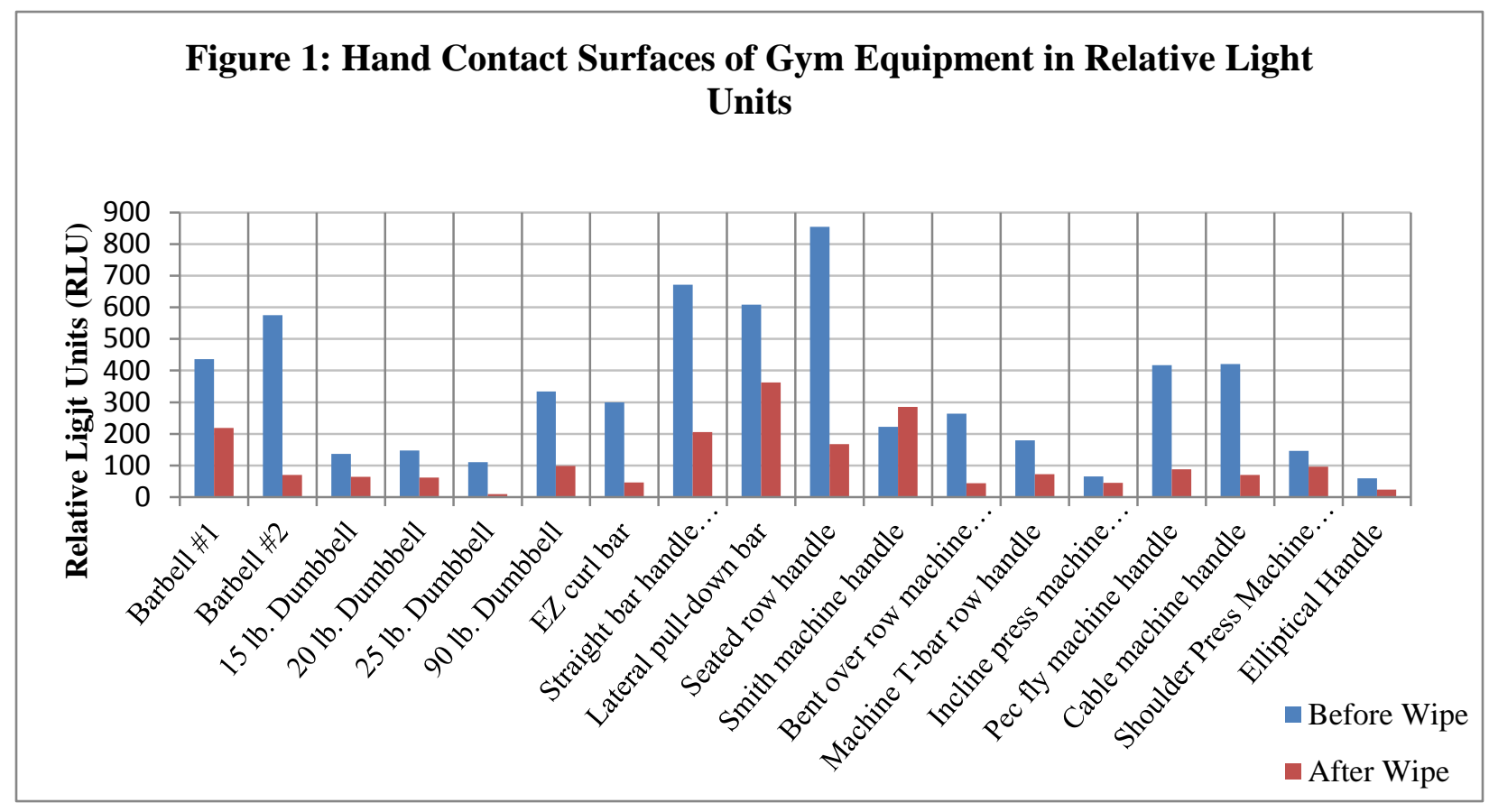




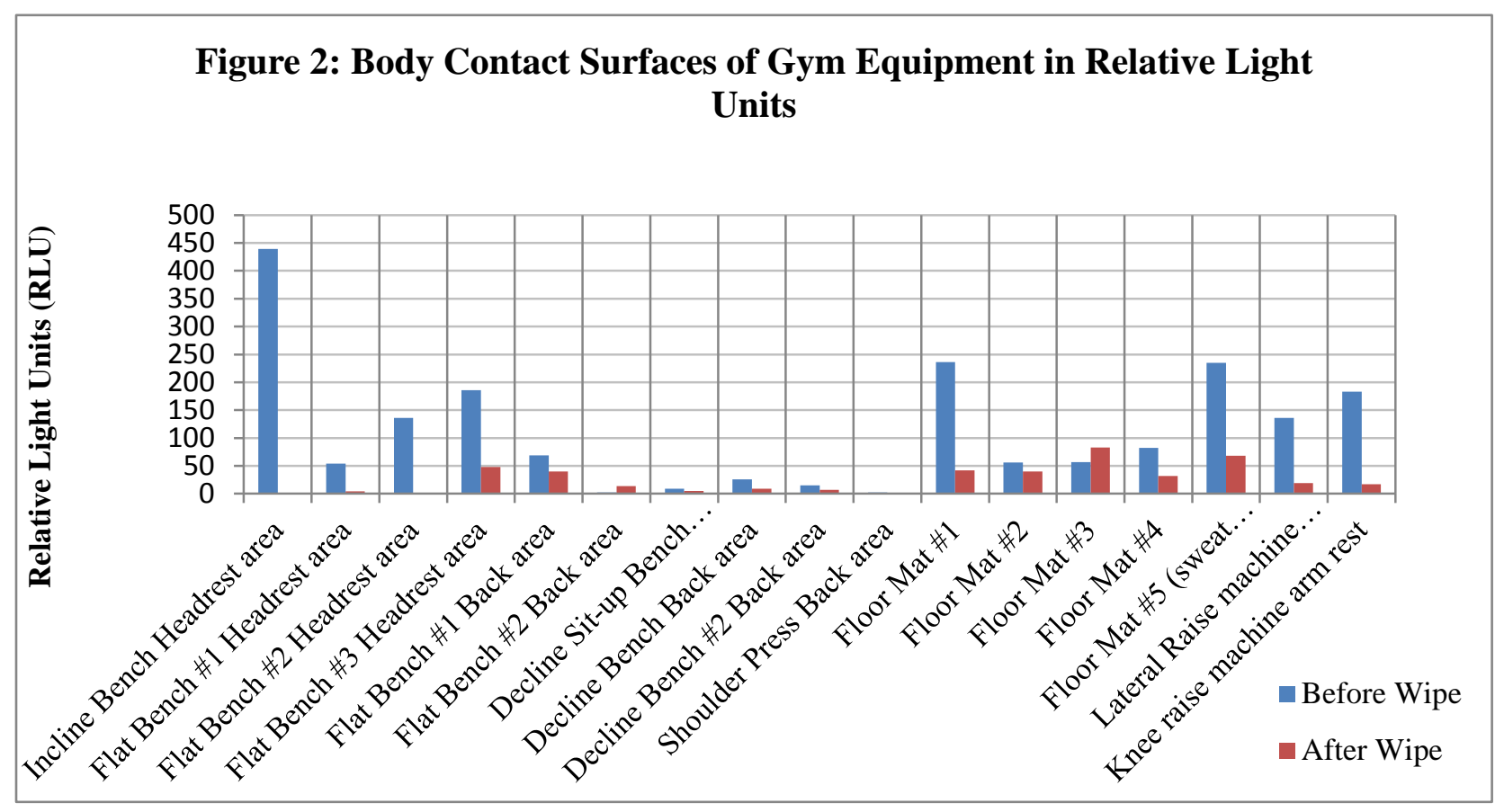

\section{Inferential Statistics}

NCSS 9 was used to analyze the inferential statistical data for the entire datasets collected. A paired sample t-test was used to analyze the variables of the data for differences before wiping and after wiping. The paired t-test performed was used to determine the differences (one sided) in ATP levels in RLUs from before and after wiping with a disinfectant wipe.

Looking at the tests for assumptions, the data represented both "cannot reject normality" and "reject normality" therefore the data is not normally distributed. Following the nonparametric Wilcoxon signed-rank test, the values were determined to be greater than 0 , therefore the null hypothesis was rejected. The probability levels for the variables (not wiped versus wiped) gave a p-value level less than 0.000001 at alpha value set at 0.05 . With the null hypothesis being rejected, the data concludes that there is a statistically significant difference in ATP levels with the use of the disinfectant wipes on gym equipment surfaces than without use of disinfectant wipes on gym equipment surfaces.

Alpha error (type 1) is highly unlikely to occur by chance with a p-value being 0.00001 at alpha set at
0.05 . Alpha set at 0.01 resulted in a p-value $=$ 0.00000 . The high power of $99.9 \%$ supports the likelihood of correctly rejecting the null hypothesis.

\section{RESULTS}

According to the information gathered and the statistical analysis' performed, the Certainty brand disinfectant wipes make a large difference in ATP levels when applied to gym contact surfaces. Mean values of RLUs for hand contact surfaces and body contact surfaces right after being used were 330 and 113 respectively; after application of disinfectant wipes, the mean values were 112 and 25 with respect.

\section{DISCUSSION}

The results as shown from the stats demonstrate a reduction in ATP levels that are derived from RLUs. For the food industry, ATP threshold levels in RLUs are considered safe at levels $0-10$, cautionary at 1130 , and a fail at 31+. Those levels are what are considered safe for food contact surfaces. According to Hygiena's healthcare benchmark, a pass is less than 50 RLUs, a cautionary is between 51 to 100 and 
a fail at 101+ RLUs. Gym equipment, however, is not a food contact surfaces nor are gyms related to a healthcare setting. Despite these ranges, there is no adopted industry standard for an acceptable RLU limit. With that being said, a comparison of contamination can be understood when the perspective data is taken into consideration.

Despite the high power from the statistical analysis of the data, all but two tests that were done on the gym contact surfaces showed a reduction in RLU levels. Details for that matter are discussed in the limitations sections below. Furthermore, the BCIT staff does routine cleaning on the floor mats and benches, but does not clean any of the handle bars and equipment, such as the dumbbells and barbells. That could be a reason why the levels were much higher for the hand contact surfaces.

The choice of clothing can also play a role in the amount of organic residue that is left behind on the surfaces. Gym patrons that were wearing normal cotton shirts against the patrons that were wearing dry-fit clothing had a different impact on the results. Sweat residue is left behind on some of the body contact surfaces when patrons wear cotton shirts, whereas sweat is absorbed when patrons wear the dry-fit clothing. The stats collected however, were not categorized for who was wearing dry-fit clothing or cotton shirts.

With respect to previous researcher studies, this study does not provide any factual evidence behind what is present on the surfaces of gym equipment, other than the general hygiene of the surfaces represented in RLUs. Microbial contamination can be present on all the equipment; however microorganisms cannot be determined unless they are swabbed and plated for a qualitative assessment. In the studies that were cited, common cases of MRSA were documented in college halls, gym facilities, and hospitals. If MRSA were present to be on the surfaces, it would require an open wound or abrasion for it to be introduced into the system. Hence, any cuts or open sores should be covered at all times when handling shared equipment to prevent an infection from occurring. In the Ryan et al. study, gym equipment was swabbed for MRSA and yielded no cultures. Because the number of MRSA carriers is rare, it can be said that only some gyms have the potential to be contaminated with MRSA. It would require a continuous succession of a gym member that is a carrier of MRSA to contaminate the gym surfaces.

Other common skin infections that can be acquired in a gym setting, such as a fungal infection, may require some conditions for disease transmission. Athlete's foot is a common fungal infection that is commonly found on the wet floors in gym showers and not on surfaces of gym equipment. Viruses also do not require any ATP to survive, thereby rendering the data collected to be ineffective for any determination of viral load. Warts generally spread from contact of contaminated surfaces rather than person-to-person contact. Palmer warts only affect the hands, therefore making it a possibility to be transmitted from gym contact surfaces. Plantar warts commonly affect the feet; therefore make it less possible to be transmitted from gym equipment but possible from gym showers. Regardless of sanitation, the influenza virus can be transmitted if a contaminated person were to spread droplets of the virus in the gym environment.

Respiration rates are higher, due to the increase of physical activity, thereby increasing changes of infection.

The National Athletic Trainers' Association provided guidelines on prevention, education, and management on soft skin tissue infections which contain a lot of information that promote the well-being of athletes. The steps on prevention should be familiarized towards the gym cleaning staff so that possible chances of infections are eliminated.

The stats give a general indication of hygiene on the equipment which can constitute it being infected with harmful pathogens. Because all the gym equipment is commonly used by everyone, it is imperative that cleaning procedures remain a standard because of how often equipment gets shared. Furthermore, hands should always be washed thoroughly after using any shared equipment.

\section{LIMITATIONS}

Limitations to this research do include the actual fact that the presence of pathogenic microorganisms and viruses are unknown to be detected on the gym contact surfaces using an ATP analyzing instrument. The Ultrasnap swabs that were used, were stored in the refrigerator. The swabs were supposed to be premoistened; however condensation formed inside the tube and some swabs were seen to be drier than others. The researcher attempted to re-moisten the swabs from the condensation inside the tubes prior to sampling. The two outliers of the test could have resulted because of the inability to pick up residues due to the lack of moisture. The surfaces of the equipment also varied between hand contact equipment and body contact equipment. Bench and 
floor mat surfaces had a much flatter surface compared to the hand contact equipment, which was cylindrical, course and unsmooth. Before testing the smith machine handle bar, it appeared to be dirty. The wet disinfectant wipe could have lifted the organic debris that was locked into the surface grooves of the handle, thereby giving a higher reading for the swab after disinfection.

\section{CONCLUSION}

The study can conclude that disinfectant wipes do make a significant difference in surface cleanliness levels. Equipment that does not undergo routine cleaning, such as the dumbbells and barbells, carry a much higher contamination rate than the body contact surfaces. Gym patrons should disinfect all body contact surfaces prior to use to reduce the risk of getting an infectious disease.

\section{RECOMMENDATIONS}

Hand contact surfaces of gym equipment were seen to carry a much larger load of contamination compared to the body contact gym equipment. Because the gym facility does not do routine cleaning on hand contact surfaces, there should be some kind of cleaning schedule made for them. People unknowingly touch their faces subconsciously. If they were to touch contaminated objects, this action can lead to infections or sicknesses.

\section{FUTURE RESEARCH SUGGESTIONS}

For future research, ATP analyzers and microbial plating should be done in conjunction with one another, to determine the relation between the two methods at a given setting. Studies on the effectiveness of dry-fit clothing against cotton clothing can also be done to determine what residual ATP levels are left behind. A study could also be conducted to determine if certain sanitizers leave a residual on the surfaces that can either inhibit the growth of microorganisms.

\section{ACKNOWLEDGEMENTS}

The author would like to thank Fred Shaw for providing all the equipment required for this study; Peter Odynsky for allowing the gym as the area of study; and Bobby Sidhu for providing direction and assistance in writing this research paper.

\section{COMPETING INTEREST}

The authors declare that they have no competing interests. 


\section{REFERENCES}

3M Microbiology. (2013). Retrieved from: http://solutions.3m.com/wps/portal/3M/en_US/Mi crobiology/FoodSafety/productapplications/three/.

BD Prepared Media. (2013). BBL RODAC Plate. Retrieved from: http://www.bd.com/ds/productCenter/210340.asp.

CDC. (2010). Cleaning \& disinfecting athletic facilities for MRSA. Retrieved on October 21, 2013 from: http://www.cdc.gov/mrsa/environment/athleticFa cilities.html

CDC. (2013a). General information about MRSA in the community. Retrieved on October 21, 2013 from: http://www.cdc.gov/mrsa/community/index.html\# q2

CDC. (2013b). Seasonal influenza: Flu basics. Retrieved on January 23, 2014, from: http://www.cdc.gov/flu/about/disease/index.htm

Canadian Center for Occupational Health and Safety. (2011) Hand washing: reducing the risk of common infection. Retrieved on October 21, 2013 from:

http://www.ccohs.ca/oshanswers/diseases/washin g_hands.html

Cohen, P.R. (2008). The skin in the gym: a comprehensive review of the cutaneous manifestations of community-acquired methicillin-resistant Staphylococcus aureus infection in athletes. Clinics in Dermatology, 26, $16-26$

Decker, C. (2010). Skin and soft tissue infections in the athlete. Dis Mon , 56, 414-421

Environmental Protection Agency. (2012). EPA's registered sterilizers, tuberculocides, and antimicrobial products against certain human public health bacteria and viruses. Retrieved from: http://www.epa.gov/oppad001/list_h_mrsa_vre.pd f.
Health Canada. (2009). Certainty Disinfectant Wipes. Retrieved from:_http://webprod5.hc-sc.gc.ca/dpdbdpp/index-eng.jsp.

Heacock, H., Crozier, V., \& Sidhu, B. (2012). Research Methods Module 5: Descriptive Statistics. British Columbia Institute of Technology.

Hintze, J. (2013). NCSS 9. [Computer software]. LLC. Kaysville, Utah, USA. Retrieved from: http://www.ncss.com.

Hygiena. (n.d.)._A comparative study of commercial ATP hygiene monitoring systems. Retrieved from: http://weberscientific.com/productresources/inserts/sillikeracomparatib811a5.pdf.

Hygiena. (2005a). SystemSURE II. Retrieved from: http://www.hygiena.net/docs/systemsureii.pdf.

Hygiena. (2005b). Ultrasnap ATP surface test. Retrieved from: http://www.hygiena.net/docs/Ultrasnap.pdf.

Hygiena. (2005c). Calibration control rod kit. Retrieved from: http://www.hygiena.net/calibration_kit.html.

Lalema. (2013). Certainty disinfectant wipes. Retrieved from: http://www.lalema.com/index.php?p=6\&i=1302\& $\mathrm{l}=\mathrm{en}$.

Lalema. (2013a). Certainty disinfectant wipes MSDS. Retrieved from: http://www.lalema.com/msds/en/certainty\%20bra nds/certainty.pdf.

Larcombe, L., Waruk, J., Schellenberg, J., \& Ormond, M. (2007). Rapid emergence of methicillin-resistant Staphylococcus aureus (MRSA) among children and adolescents in northern Manitoba, 2003-2006. Canada Communicable Disease Report, 33(2)

Leon, M., \& Albrecht, J., (2007). Comparison of adenosine triphosphate (ATP) bioluminescence and aerobic plate counts (APC) on plastic cutting boards. Journal of Foodservice, 18, 145-152.

Miller, L.G., \& Diep, B.A. (2008). Colonization, fomites, and virulence: Rethinking the pathogenesis of community-acquired methicillinresistant Staphylococcus aureus infection. Clinical Infectious Diseases, 46, 752-760 
New York Times. (August 2, 2010). Be sure exercise is all you get at the gym. Retrieved on October 7, 2013 from:

http://www.nytimes.com/2010/08/03/health/03bro d.html?_r=4\&adxnnl=1\&adxnnlx=1381972817pOZH14nSdmaK48OQyMIQZA\&

Pinto, F., Hiom, S., Girdlestone, S., \& Maillard, J.Y. (2009). Evaluating the effectiveness of commercially available contact plates for monitoring microbial environments. Letters In Applied Microbiology, 48, 379-382.

Rockwell, F., Goh, S.H., Al-Rawahi, G., Hoang, L., Isaac-Renton, J., \& et al. (2005). A report on the emergence of community-acquired methicillinresistant Staphylococcus aureus (CA-MRSA) in British Columbia. Retrieved from www.canada.com/vancouversun/news/extras/MR SA_report.pdf

Ryan, K.A., Ifantides, C., Bucciarelli, C., Saliba, H., Tuli, S., Black, E., \& Thompson, L.A. (2011). Are gymnasium equipment surfaces a source of staphylococcal infections in the community? Association for Professionals in Infection Control and Epidemiology, 39, 148-150
Shukla, S.K. (2006). CA-MRSA triangulation: Virulent strains, susceptible hosts, and contaminated environments. Wisconsin Medical Journal, 105(6), 21-23

Skov, R., Christiansen, K., Dancer, S.J., \& et al. (2012). Update on the prevention and control of community-acquired methicillin-resistant Staphylococcus aureus (CA-MRSA). International Journal of Antimicrobial Agents, 39, 193-200

Stanforth, B., Krause, A., Starkley, C., \& Ryan, T.J. (2010). Prevalence of community-associated methicillin-resistant Staphylococcus aureus in high-school wrestling environments. Journal of Environmental Health, 72(6),12-16

Tonn, K., \& Ryan, T.J., (2012). Communityassociated methicillin-resistant Staphylococcus aureus in college residential halls. Journal of Environmental Health, 75(6), 44-49

Zinder, S.M., Basler, R.S., Foley, J., Scarlata, C., \& Vasily, D.B. (2010). National athletic trainers' association position statement: skin diseases. Journal of Athletic Training, 45(4), 411-42 